\title{
Response to postoperative radiotherapy as a prognostic factor for patients with low-grade gliomas
}

\author{
MICHAL SPYCH ${ }^{1,2}$, LESZEK GOTTWALD $^{3}$, EMILIA JESIEŃ-LEWANDOWICZ ${ }^{1,2}$, \\ SŁAWOMIR SZTAJER ${ }^{4}$ and JACEK FIJUTH ${ }^{1,2}$ \\ ${ }^{1}$ Radiotherapy Department, Chair of Oncology, Medical University of Lodz; ${ }^{2}$ Teleradiotherapy Department, \\ Regional Center of Oncology, Copernicus Memorial Hospital; ${ }^{3}$ Palliative Care Unit, Chair of Oncology, \\ Medical University of Lodz; ${ }^{4}$ Radiology Department, Medical University of Lodz, Lodz, Poland
}

Received January 2, 2012; Accepted March 5, 2012

DOI: $10.3892 / \mathrm{ol} .2012 .759$

\begin{abstract}
Due to the favorable natural history in patients with low-grade gliomas (LGGs), there is no consensus on the treatment strategy following maximal safe surgical resection. A number of studies have been conducted to identify prognostic factors in patients treated for LGG. The present study evaluated the treatment outcomes as well as prognostic factors and their impact on overall survival (OS) and disease-free survival (DFS). We retrospectively reviewed 30 consecutive patients treated for LGG at the Department of Radiotherapy from February 2008 to July 2011. The patients underwent surgical intervention and postoperative radiotherapy. The response to radiotherapy was evaluated from six to eight weeks after the end of treatment using MRI analysis. Kaplan-Maier analysis was used for OS and DFS estimation. The endpoint was mortality as a result of any cause. Within a median follow-up of 21.8 months, 9 patients $(30 \%)$ with disease progression were reported. The two- and five-year DFS and OS was 85.2 and $68.3 \%$ for DFS, and 84.3 and $63.4 \%$ for OS, respectively. The response to radiotherapy, evaluated in an MRI study, was found to be highly correlated with OS ( $<<0.0001)$. We also observed a significantly higher OS in patients with disease progression treated with salvage chemotherapy after the end of radiotherapy $(\mathrm{p}=0.08)$. Improved outcome among patients with LGG may be predicted by response to radiotherapy evaluated by MRI following termination of treatment.
\end{abstract}

\section{Introduction}

Low-grade gliomas (LGGs) have a relatively slow growth rate. Adjuvant treatment following maximal safe surgical resection for patients with LGG remains controversial due to the favorable natural history of the disease. A survival benefit in

Correspondence to: Dr Michal Spych, Radiotherapy Department, Chair of Oncology, Medical University of Lodz, Paderewskiego 4, Lodz 93-509, Poland

E-mail: michal.spych@umed.lodz.pl

Key words: low-grade gliomas, radiotherapy, prognostic factors patients treated with aggressive surgical resection and postoperative radiotherapy has not been demonstrated in prospective clinical trials. The majority of retrospective studies demonstrated improved outcomes in groups of patients with total or subtotal resection (1-3). However, even with aggressive surgical procedures, total resection is rarely achieved. This is due to the tumor size, location in critical regions of the brain and the diffusely infiltrative nature of these tumors.

In general, the majority of patients undergo surgery at the time of symptom presentation. Consequently, it is difficult to establish the histopathological diagnosis and grade, and to determine further treatment strategies. Three phase III trials investigated the role of radiotherapy in patients with LGGs who underwent surgical resection. In the EORTC 22845 multi-institutional study, patients were randomized to receive postoperative radiotherapy of $54.0 \mathrm{~Gy}$, or radiotherapy at the time of progression. A statistically significant improvement in median progression-free survival (PFS), 5.3 versus 3.4 years $(\mathrm{p}<0.0001)$, was found in the group of patients treated with early radiotherapy compared to patients treated with radiotherapy at progression. There was no difference in the median OS in the study, 7.4 versus 7.2 years in the group of patients with early versus late radiotherapy $(\mathrm{p}=0.8)$ (4). In the subsequent study (EORTC 22844), the radiation dose was established in postoperative patients, who were randomized to receive 45.0 or $59.4 \mathrm{~Gy}$. There were no statistically significant differences in 5-year OS, 58 versus 59\%, and PFS, 47 versus 50\%, in patients who received 45.0 and $59.4 \mathrm{~Gy}$, respectively (5). In the third study, patients were again randomized to radiotherapy at a low dose of 50.4 Gy or a high dose of 64.8 Gy. There were no statistically significant differences in OS and PFS, but grade 3-5 neurotoxicity was observed in $5 \%$ of patients treated with a high-dose of radiotherapy compared to $2.5 \%$ of patients in the low-dose regimen (6). Several studies have been conducted to identify prognostic factors in patients treated for LGG $(1,3,7,8)$. The age at the time of diagnosis, performance status, histological subtype, tumor site and size, type of symptoms at diagnosis, the duration of disease symptoms prior to diagnosis and extent of resection have been proposed as prognostic factors for PFS and OS. However, in many of these retrospective studies, the small number of patients and the heterogeneity of treatments limited the analysis of prognostic factors. 
Pignatti et al (9) conducted an analysis, which was based on data from EORTC 22844 and 22845 studies, and presented a scoring system to identify patients with a low and high risk. The multivariate analysis showed that patients $>40$ years, with astrocytoma histopathology, a tumor size of $\geq 6 \mathrm{~cm}$, tumor crossing the midline, as well as occurrence of neurological deficits, were correlated with worse survival (9). In our retrospective study, the treatment outcomes of patients with LGG were presented. Potential prognostic factors were evaluated as well as their impact on DFS and OS.

\section{Patients and methods}

Patients. A total of 30 patients who underwent tumor excision and adjuvant radiotherapy due to diagnosis of brain tumor were enrolled in this retrospective study. The patients underwent radical radiotherapy between February 2008 and July 2011 at the Radiotherapy Department of the Medical University of Lodz, Poland. There were 16 males (53\%) and 14 females $(47 \%)$. The male to female ratio was 1.1:1. The age of patients fluctuated between 20 and 72 years [mean 41.4; median 40.5; 95\% confidence interval (CI 95\%) 36.9-45.9; standard deviation (SD) 12.0]. In the analyzed group, the performance status of patients was evaluated using the Karnofsky Performance Scale (KPS) index. There were three patients (10\%) classified at $100 \%$ KPS. Fourteen (47\%) and 8 patients $(27 \%)$ were classified at 90 and $80 \%$ KPS, respectively. Five patients (17\%) were classified at $70 \%$ KPS. The duration of disease symptoms prior to the start of treatment ranged from 1 to 107 days. The mean duration of disease symptoms was $19.0 \pm 30.9$ days (median 6.0, CI 95\% 7.1-31.0). Tumor limited to one lobe was reported in 20 patients (67\%). In seven cases (23\%) the tumor was localized within two or three lobes. In two (7\%) and one (3\%) patients the tumor was limited to the midline zone and cerebellum, respectively. The tumor size (the largest diameter defined in millimeters, measured by preoperative CT or MRI) ranged from 14 to $102 \mathrm{~mm}$ (mean $69.9 \pm 35.2$; median 85; CI 95\% 56.5-83.3).

The tumor tissue collected during surgery allowed a histopathological diagnosis to be determined in all patients, and became a basis for planning the further treatment strategy. According to tumor histology, there were 14 astrocytomas (47\%), 3 oligodendrogliomas (10\%), 8 mixed tumors (26\%) and 5 gemistocytic astrocytomas (17\%). Five patients (17\%) underwent a total tumor excision. All patients were enrolled in the study after total excision due to high risk of surgical treatment failure (patients $>40$ years, astrocytoma histopathology, tumor size of $\geq 6 \mathrm{~cm}$, tumor crossing the midline as well as occurrence of neurological deficits) (9). In total, 19 (63\%) and 6 patients (20\%) underwent subtotal excision and biopsy, respectively. The mean size of the residual tumor following subtotal resection or biopsy was $51.5 \pm 18.6 \mathrm{~mm}$ (median 47.5; CI 95\% 44.5-58.4). In the group of patients who underwent total and subtotal tumor excision 3 recurrences (10\%) and 3 cases of disease progression (10\%), respectively, were observed prior to the start of adjuvant therapy. Four patients (13\%) underwent tumor re-excision, which was defined as the subtotal in all cases. No adjuvant chemotherapy was administered following surgery. Patient and tumor characteristics are shown in Table I.
Table I. Patient characteristics and tumor details.

\begin{tabular}{lr}
\hline Analysed factors & $\mathrm{N}(\%)$ \\
\hline Gender & \\
Males & $16(53)$ \\
Females & $14(47)$ \\
Age (years) & \\
$<40$ & $14(47)$ \\
$\geq 40$ & $16(53)$ \\
Performance status & \\
$\geq 90 \%$ & $17(57)$ \\
$<90 \%$ & $13(43)$ \\
Duration of disease symptoms (days) & \\
$\geq 19$ & $20(67)$ \\
$<19$ & $10(33)$ \\
Tumor site & \\
One lobe & $20(67)$ \\
Two or three lobes & $7(23)$ \\
Midline zone & $2(7)$ \\
Cerebellum & $1(3)$ \\
Tumor size (cm) & \\
$<3$ & $3(10)$ \\
$3-5$ & $13(43)$ \\
$>5$ & $14(47)$ \\
Histopathology & \\
Astrocytoma & $14(47)$ \\
Oligodendroglioma & $3(10)$ \\
Mixed tumor & $8(26)$ \\
Gemistocytic astrocytoma & $5(17)$ \\
Residual tumor & \\
Yes & $25(83)$ \\
No & $5(17)$ \\
Response to radiotherapy & \\
Complete remission & $10(33)$ \\
Stabilization & $17(57)$ \\
Disease progression & $3(10)$ \\
\hline & \\
&
\end{tabular}

The study was approved by the ethics committee of the Medical University of Lodz. Consent was obtained from the patients or from a family member.

Radiotherapy. External beam radiotherapy was delivered using a linear accelerator (6 MV photons). Precise patient immobilization and reproducibility of head positioning was achieved by using thermoplatic masks. The treatment volumes as well as critical organs (optic nerves and optic chiasm, lenses and brain stem) and radiation dose were defined using the 'Eclipse Varian' computer planning system. The tumor or tumor bed demonstrated on preoperative T1- and T2-weighted MRI sequences with a margin of $1.5 \mathrm{~cm}$, included in clinical target volume (CTV), were treated. The three-dimensional conformal technique was used in each case. Treatment 
volumes were defined according to the 50 and 62 International Commission on Radiation Units and Measurements (ICRU) reports. The target volume was covered by the $95 \%$ isodose. The daily radiation dose was $1.8 \mathrm{~Gy}$ increasing to a total radiation dose of 54.0 Gy in 30 fractions. In the group of patients with gemistocytic astrocytoma histopathology, the total dose was escalated to $60 \mathrm{~Gy}$, with $2.0 \mathrm{~Gy}$ per fraction. Treatment was administered five days per week for five weeks. The correctness of treatment plan realization was checked on a simulator, where treatment fields were transmitted on the patient's skin, and saved in the computer system simultaneously. Treatment field set-up repeatability was evaluated through simulator and accelerator image fusion and geometric error measurement.

Follow-up. Patients were followed up after the end of radiotherapy. A general and neurological examination was evaluated during every follow-up visit, as well as a brain MRI study. Patients were evaluated for disease progression as well as treatment-related toxicity. The treatment-related toxicity was evaluated according to the RTOG/EORTC classification. The response to radiotherapy was evaluated from six to eight weeks following the end of treatment using MRI study. Complete remission was achieved in 10 patients $(33 \%)$. Disease progression and stabilization were observed in 3 (10\%) and 17 patients (57\%), respectively. In 9 patients $(30 \%)$, disease progression was reported during the whole follow-up period. The disease progression was confirmed in a brain MRI study. The tumor size after progression ranged from 20 to $106 \mathrm{~mm}$ (mean 90.8 \pm 27.2 ; median 85; CI 95\% 80.3-101.2). Three patients with disease progression underwent re-excision defined as subtotal resections.

The histological progression of malignancy to grade III (anaplastic variants), according to WHO scale, was reported in two patients following re-excision. One patient qualified for stereotactic radiosurgery as salvage retreatment. A dose of 12.0 Gy radiation in $80 \%$ isodose was delivered. Chemotherapy as a salvage treatment was used in a group of six patients with disease progression. Three patients received temozolomide (TMZ), and the remaining three patients received the PCV regimen (procarbazine, lomustine and vincristine). Of 5 patients treated with chemotherapy, only one did not receive six cycles. One patient treated with the PCV regimen received 4 cycles due to general and neurological deterioration. In four patients treated with chemotherapy, further progression was reported in imaging studies. Partial and complete regression were reported in two patients.

Statistical analysis. In the present retrospective study, OS and DFS for all patients have been estimated. The Kaplan-Meier analysis was used for OS and DFS estimation. The end-point of the analysis was mortality as a result of any cause. The Chi-square test was used to determine whether the distribution of prognostic variables was significantly different. Comparisons of survival curves were made using the log-rank test. Clinical prognostic factors including age, performance status, duration of the disease symptoms, tumor site, postoperative margin status, tumor size, histopathological tumor type, recurrence after surgery, response to adjuvant radiotherapy and use of chemotherapy were included in the statistical analysis. $\mathrm{P}<0.05$ was considered to indicate a statistically significant difference.

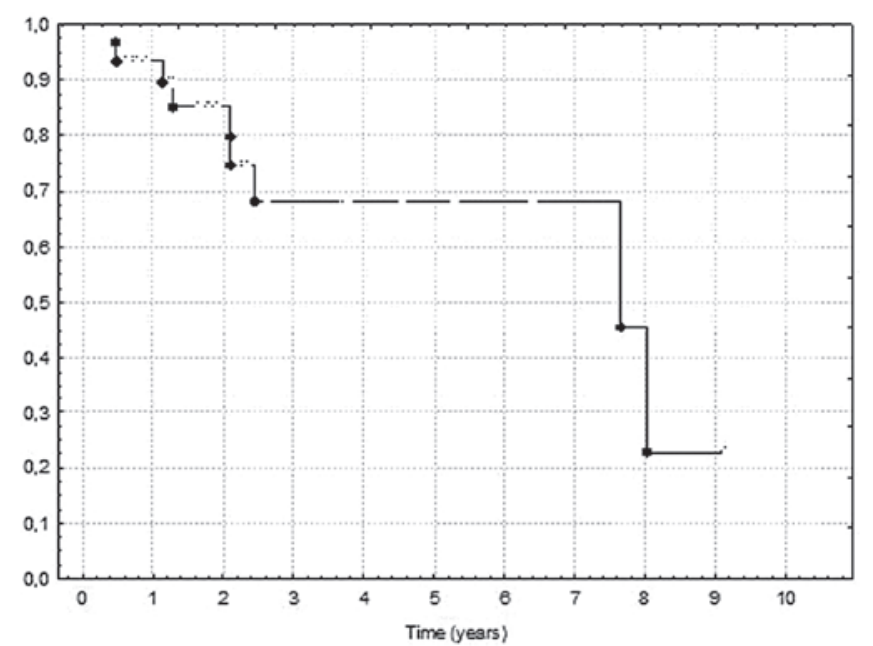

Figure 1. Disease-free survival for the entire group of patients included in the statistical analysis.

\section{Results}

Disease progression and clinicopathological characteristics. Within a median follow-up of 21.8 months (range, 3.9-52.6) there were 9 patients (30\%) reported to have disease progression. In the analyzed group of patients, the 2- and 5-year DFS was 85.2 and $68.3 \%$, respectively. The mean DFS was 33.6 months (range, 5.6-107.5; median 24.8; 95\% CI 23.3-43.9; SD 27.6). The 2- and 5-year OS in the analyzed group of patients was 84.3 and $63.4 \%$, respectively. The mean OS was 36.2 months (range, 5.6-107.5; median 26.9; 95\% CI 25.4-46.8; SD 28.6). The 2- and 5-year DFS and OS are shown in Figs. 1 and 2, respectively. In the present analyzed group of patients, the age, performance status, duration of disease symptoms and tumor site, as well as tumor size and histopathological tumor type, did not have any impact on DFS and OS. The residual tumor following surgery did not show any negative values in respect to OS ( $\mathrm{p}=0.7)$, but we noted a trend towards statistically significant longer OS in the group of patients who underwent re-excision due to recurrence before radiotherapy had been initiated $(\mathrm{p}=0.03)$.

Overall survival in respect to response to radiotherapy. In the statistical analysis, we reported that response to radiotherapy evaluated from 6 to 8 weeks after treatment termination was highly correlated with OS. The mean OS in the group of patients with noted complete remission was 4.03 years compared to 2.9 years in the group with disease stabilization. The mean survival in the group of patients with progression in the period of 6-8 weeks following radiotherapy termination was 8.3 months. The p-value in the log-rank analysis was $<0.0001$. The OS in responders and non-responders is shown in Fig. 3. In the presented analysis we observed a statistically significant higher OS in patients with disease progression after the end of radiotherapy treated with salvage chemotherapy $(\mathrm{p}=0.08)$. The mean OS of patients treated with salvage chemotherapy was 3.8 years compared to 2.8 years in the group of patients without the treatment. Late toxicity was not assessed in our group of patients due to the retrospective nature of the study. 


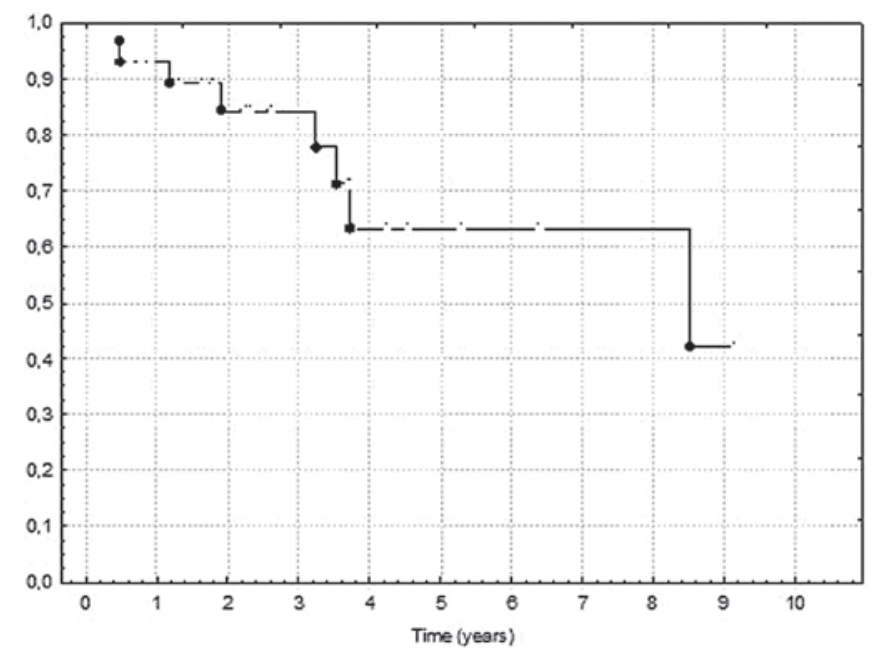

Figure 2. Overall survival for the entire group of patients included in the statistical analysis.

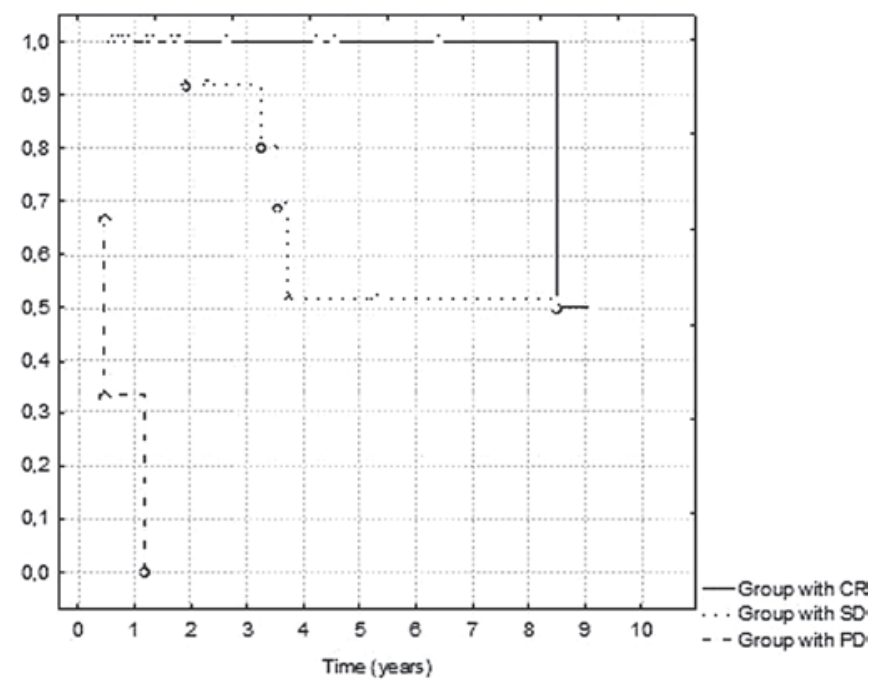

Figure 3. Overall survival in respect to response to radiotherapy. CR, complete remission; SD, disease stabilization; $\mathrm{PD}$, disease progression.

For those patients who were alive without any active tumor at the last follow-up, the quality of life was determined to be extremely good, without evidence of postradiation necrosis in imaging studies.

\section{Discussion}

The prognosis for patients with LGG is favorable, and long survival is expected in this group of patients. In the three largest randomized trials, EORTC 22844, EORTC 22845 and the NCCTG trial, the 5-year OS ranged from 58 to $72 \%$. In the present study, the 5-year OS and DFS was 63.4 and $68.3 \%$, respectively. The treatment results are in agreement with previously reported studies (4-6). The role of the extent of surgical excision in the treatment of patients with LGG is controversial. Extent of surgical excision was identified as a prognostic factor in a number of studies $(1,3,4-6,10,11)$. However, it is worth noting that some authors reported that the extent of surgical excision was not correlated with DFS and OS $(12,13)$. It was postulated that extensive resection was possible to conduct due to the limited size and superficial localization of the tumor. However, our results showed the extent of surgical excision did not have any impact on DFS and OS rates. LGGs are infiltrative tumors with microscopic invasion beyond the radiographic margins, thus, gross total resection is rarely achieved. In total, 25 patients included in our study received immediate radiotherapy with a total dose of $54.0 \mathrm{~Gy}$ in 30 fractions, and the remaining five patients with gemistocytic astrocytoma histopathology received a total dose of $60 \mathrm{~Gy}$. The EORTC 22845 study compared immediate radiotherapy with treatment after recurrence. OS and time to progression were the primary end points. In this study, immediate postoperative irradiation increased the median PFS, but not OS (4). The EORTC study conducted a detailed analysis of prognostic factors for OS based on the 22844 and 22845 studies. Factors that negatively affected survival were age $>40$ years, astrocytoma histology, maximum tumor diameter $\geq 6 \mathrm{~cm}$, tumor crossing the corpus callosum and the presence of a neurological deficit prior to surgery $(4,5)$.

In the present retrospective study, the patients had indications for radiotherapy according to the negative prognostic factors that were defined in the EORTC studies. These factors were included in the statistical analysis. The majority of studies found some correlation between survival and signs and symptoms at the time of presentation. A number of studies found that the presence of neurological deficits as well as the poor performance status of the patients were negative prognostic factors $(3,7,10,11)$. In one study, the authors noted the favorable outcomes in a group of patients presenting with seizures as the only symptom of disease (3). In the present analysis patients' KPS, and the duration of disease symptoms were evaluated. Our results did not show an association between these values and treatment outcomes. These three factors (performance status, presence of seizures and neurological deficits) are connected. When neurological or other functional deficits are observed, a decrease in the performance status is reported. Pignatti et al revealed a close relationship between the presence of seizures and presence of other neurological symptoms (9). This association was also observed in a study in which epilepsy was associated with a better outcome if this was the only symptom at presentation. When other symptoms were presented, seizures were no longer a positive prognostic factor (14).

Age is the fundamental prognostic factor for survival of patients with LGGs $(3-5,7,10,11,13-15)$. The elderly patients had a worse prognosis. In one study, a linear relationship between age and patient prognosis was reported (7). A cut-off point of 40 years was selected in the majority of the abovementioned studies. In the present group of patients the mean age was 41.4 years, median 40.5 and $95 \%$ CI was 36.9-45.9. Our results did not confirm a significant association between age and prognosis of patients with LGGs. This finding was probably due to the small number of patients in the analysis, and the non-parametric distribution of this value. We also did not find a significant correlation between tumor histology subtype and DFS or OS. This was probably due to the small number of patients included in the statistical analysis and the unequal distribution of histological subgroups. 
In the majority of studies, patients with oligodendrogliomas or mixed oligoastrocytic tumors had a more favorable prognosis than patients with pure astrocytic histology $(3-5,8,9)$. In the present study, the response to radiotherapy following treatment termination was highly correlated with higher OS rate. The mean OS in the group of patients with complete remission was 4.03 years compared to 2.9 years in the group with disease stabilization. The mean survival in the group of patients with progression in the period of 6-8 weeks following radiotherapy termination was 8.3 months. The p-value in the log-rank analysis was $<0.0001$. Few data are available in the literature describing the correlation between response of LGGs to radiotherapy and treatment outcomes. Bauman et al (16) analyzed symptomatic improvement and PFS as a function of radiographic response in a group of 21 patients with LGGs treated with postoperative radiotherapy. The authors reported clinical improvement and a $\geq 50 \%$ decrease in the maximum tumor cross-sectional area in 11 patients (52\%). More partial responders improved symptomatically than non-responders, but there was no statistically significant correlation between radiographic response and PFS. The median time to maximum response was 2.8 months following treatment termination. The median time to progression measured from the start of radiotherapy was 4.8 years, and the 5-year PFS rate was $43 \%$ (16). The results of this study suggest that LGGs are moderately radioresponsive tumors. These results were confirmed by other authors $(17,18)$. Eyre et al reported an $80 \%$ rate of response to radiotherapy (17), whereas results of another study found the rate of response to radiotherapy to be $46 \%$ (18). In the EORTC 22845 and EORTC 22844 studies postoperative computed tomography scans were not routinely performed, thus, the residual amount of tumor was not assessed, and the response to radiotherapy was not evaluated $(4,5)$. For patients with LGGs suffering from tumor progression, the optimal management is unclear. In the present study, we observed a statistically significant higher OS in patients treated with salvage chemotherapy due to disease progression after the end of radiotherapy. In high-risk patients, the adjuvant chemotherapy after postoperative radiotherapy has been explored in a large randomized RTOG trial. Patients were randomized to postoperative radiotherapy with or without adjuvant PCV chemotherapy. Patients were stratified by age, histology, performance status and presence/absence of contrast enhancement on postoperative MRI studies. The initial analysis following a median follow-up of $>4$ years showed that adjuvant chemotherapy did not translate into improved outcome in high-risk patients (19). Response to treatment and prognosis may vary in patients with LGGs.

Several phase II studies of PCV or TMZ chemotherapy regimens in the treatment of new, progressive or recurrent LGGs have been performed (20-26). A similar response rate was observed for astrocytomas and oligodendrogliomas $(20,25,26)$. Chemotherapy was effective in previously treated and untreated patients $(21,22,26)$. Although oligodendrogliomas with deletion of chromosome $1 p$ and $19 q$ may be more sensitive to treatment $(23,28)$, tumors without these deletions were also responsive to chemotherapy (27). The natural history of oligodendroglial tumors is more protracted compared with astrocytic tumors. Furthermore, oligodendrogliomas show a higher sensitivity to chemotherapy. In particular, pure oligodendrogliomas with a loss of heterozygosity on chromosomes
$1 \mathrm{p} / 19 \mathrm{q}$ have been identified as tumors with a much more favorable natural history, irrespective of treatment (29). In the present study, patients who qualified for salvage chemotherapy were young, without neurological deficits, and with good general condition. These are commonly known positive prognostic factors that are associated with better treatment outcomes. The small number of patients, heterogeneity and retrospective character of the study does not allow for the generalization of these results to the whole population.

Additional prospective studies are required for a more robust description of the association between the response to postoperative radiotherapy and treatment outcomes. If the evidence for the existence of this association was demonstrated, it would be possible to identify high-risk patients with indications for adjuvant chemotherapy, or escalation of radiation dose. Thus, these issued should be investigated in future studies.

\section{References}

1. Berger MS, Deliganis AV, Dobbins J and Keles GE: The effect of extent of resection on recurrence in patients with low grade cerebral hemisphere gliomas. Cancer 74: 1784-1791, 1994.

2. Claus EB, Horlacher A, Hsu L, et al: Survival rates in patients with low-grade glioma after intraoperative magnetic resonance image guidance. Cancer 103: 1227-1233, 2005.

3. Leighton C, Fisher B, Bauman G, et al: Supratentorial low-grade in adults: an analysis of prognostic factors and timing of radiation. J Clin Oncol 15: 1294-1301, 1997.

4. Van den Bent MJ, Afra D, de Witte O, et al: Long-term efficacy of early versus delayed radiotherapy for low-grade astrocytoma and oligodendroglioma in adults: the EORTC 22845 randomized trial. Lancet 366: 985-990, 2005.

5. Karim AB, Maat B, Hatlevoll R, et al: A randomized trial on dose-response in radiation therapy of low-grade cerebral glioma: European Organization for Research and Treatment of Cancer (EORTC) Study 22844. Int J Radiat Oncol Biol Phys 36: 549-556, 1996.

6. Shaw E, Arusell R, Scheithauer B, et al: Prospective randomized trial of low versus high-dose radiation therapy in adults with supratentorial low-grade glioma: initial report of a North Central Cancer Treatment Group/ Radiation Therapy Oncology Group/ Eastern Cooperative Oncology Group study. J Clin Oncol 20: 2267-2276, 2002

7. Lote K, Egeland T, Hager B, et al: Survival, prognostic factors, and therapeutic efficacy in low-grade glioma: a retrospective study in 379 patients. J Clin Oncol 15: 3129-3140, 1997.

8. Janny $\mathrm{P}$, Cure $\mathrm{H}$, Mohr M, et al: Low grade supratentorial astrocytomas: management and prognostic factors. Cancer 73: 1937-1945, 1994

9. Pignatti F, van den Bent M, Curran D, et al: Prognostic factors for survival in adult patients with cerebral low-grade glioma. J Clin Oncol 20: 2076-2084, 2002.

10. Nicolato A, Gerosa MA, Fina P, Iuzzolino P, Giorgiutti F and Bricolo A: Prognostic factors in low-grade supratentorial astrocytomas: a uni-multivariate statistical analysis in 76 surgically treated patients. Neurosurgery 44: 208-223, 1995.

11. Soffietti R, Chiò A, Giordana MT, Vasario E and Schiffer D: Prognostic factors in well-differentiated cerebral astrocytomas in the adult. Neurosurgery 24: 686-692, 1989.

12. Shibamoto Y, Kitakabu Y, Takahashi M, et al: Supratentorial low-grade astrocytoma. Correlation of computed tomography findings with effect of radiation therapy and prognostic variables. Cancer 72: 190-195, 1993.

13. Vecht CJ: Effect of age on treatment decisions in low-grade glioma. J Neurol Neurosurg Psychiatry 56: 1259-1264, 1993.

14. van Veelen ML, Avezaat CJ, Kros JM, van Putten W and Vecht C: Supratentorial low grade astrocytoma: prognostic factors, dedifferentiation, and the issue of early versus late surgery. J Neurol Neurosurg Psych 64: 581-587, 1998.

15. Bauman G, Fischer B, Walting C, Cairncross JG and Macdonald D: Adult Supratentorial low-grade gliomas: long experience at a single institution. J Radiat Oncol Biol Phys 75: 1401-1407, 2009. 
16. Bauman G, Pahapill P, Macdonald D, Fisher B, Leighton C and Cairncross G: Low grade glioma: a measuring radiographic response to radiotherapy. Can J Neurol Sci 26: 18-22, 1999.

17. Eyre HJ, Crowley JJ, Townsend JJ, et al: A randomized trial of radiotherapy versus radiotherapy plus CCNU for incompletely resected low-grade gliomas: a Southwest Oncology Group study. J Neurosurg 78: 909-914, 1993.

18. Lunsford LD, Somaza S, Kondziolka D and Flickenger JC: Survival after stereotactic biopsy and radiation of cerebral non pilocytic astrocytoma. J Neurosurg 82: 523-529, 1995.

19. Shaw EG, Berkey B, Coons SW, et al: Initial report of Radiation Therapy Oncology Group (RTOG) 9802: Prospective studies in adult low-grade glioma (LGG). ASCO 2006 Annual Meeting Proceedings. J Clin Oncol 24: Abstr 1500, 2006.

20. Quinn JA, Reardon DA, Friedman AH, et al: Phase II trial of temozolomide in patients with progressive low-grade glioma. J Clin Oncol 21: 646-651, 2003.

21. van den Bent MJ, Taphoorn MJ, Brandes AA, et al: Phase II study of first-line chemotherapy with temozolomide in recurrent oligodendroglial tumors: the European Organization for Research and Treatment of Cancer Brain Tumor Group study 26971. J Clin Oncol 21: 2525-2528, 2003

22. van den Bent MJ, Chinot O, Boogerd W, et al: Second-line chemotherapy with temozolomide in recurrent oligodendroglioma after PCV (procarbazine, lomustine and vincristine) chemotherapy: EORTC Brain Tumor Group phase II study 26972. Ann Oncol 14: 599-602, 2003.
23. Hoang-Xuan K, Capelle L, Kujas M, et al: Temozolomide as initial treatment for adults with low-grade oligodendrogliomas or oligoastrocytomas and correlation with chromosome $1 \mathrm{p}$ deletions. J Clin Oncol 22: 3133-3138, 2004.

24. Buckner JC, Gesme D Jr, O'Fallon JR, et al: Phase II trial of procarbazine, lomustine, and vincristine as initial therapy for patients with low-grade oligodendroglioma or oligoastrocytoma: efficacy and associations with chromosomal abnormalities. J Clin Oncol 21: 251-255, 2003.

25. Brada M, Viviers L, Abson C, et al: Phase II study of primary temozolomide chemotherapy in patients with WHO grade II gliomas. Ann Oncol 14: 1715-1721, 2003.

26. Pace A, Vidiri A, Galiè E, Carosi M, Telera S, Cianciulli AM, et al: Temozolomide chemotherapy for progressive low-grade glioma: clinical benefits and radiological response. Ann Oncol 14: 1722-1726, 2003.

27. Stege EM, Kros JM, de Bruin HG, et al: Successful treatment of low-grade oligodendroglial tumors with a chemotherapy regimen of procarbazine, lomustine, and vincristine. Cancer 103: 802-809, 2005

28. Chahlavi A, Kanner A, Peereboom D, Staugaitis SM, Elson P and Barnett G: Impact of chromosome 1p status in response of oligodendroglioma to temozolomide: preliminary results. J Neurooncol 61: 267-273, 2003.

29. Jenkins RB, Blair H, Ballman KV, et al: A $\mathrm{t}(1 ; 19)(\mathrm{q} 10 ; \mathrm{p} 10)$ mediates the combined deletions of $1 \mathrm{p}$ and $19 \mathrm{q}$ and predicts a better prognosis of patients with oligodendroglioma. Cancer Res 66: 9852-9861, 2006 